\title{
BOUNDED COHOMOLOGY OF CERTAIN GROUPS OF HOMEOMORPHISMS
}

\author{
SHIGENORI MATSUMOTO AND SHIGEYUKI MORITA
}

\begin{abstract}
We consider the condition when bounded cohomology injects into ordinary cohomology and prove the vanishing of bounded cohomology of the group of all compactly supported homeomorphisms of $\mathbf{R}^{n}$.
\end{abstract}

Introduction. In this note we consider relations among bounded cohomology, ordinary real cohomology and $l^{1}$ homology of spaces or groups. In particular we present a necessary and sufficient condition under which bounded cohomology injects into ordinary cohomology and by using it prove the vanishing of bounded cohomology and $l^{1}$ homology of $\mathrm{Homeo}_{K} \mathbf{R}^{n}$, the group of all homeomorphisms of $\mathbf{R}^{n}$ with compact support. We also determine the second bounded cohomology of $\mathrm{SL}_{2} \mathbf{R}$.

1. Bounded cohomology. Let us quickly review the theory of bounded cohomology developed by Gromov [2] (see also Brooks [1] and Mitsumatsu [5]). Let $X$ be a topological space and let $\mathscr{C}_{*}(X)=\left\{C_{q}(X), \partial_{q}\right\}$ be the singular chain complex of $X$ with real coefficients. Define a norm on $C_{q}(X)$ by $\left\|\sum_{i=1}^{n} a_{i} \sigma_{i}\right\|=\sum_{i=1}^{n}\left|a_{i}\right|$. The differentials $\partial_{q}$ are then bounded linear operators.

Let $\mathscr{C}_{*}^{l_{1}}(X)=\left\{C_{q}^{l_{1}}(X), \partial_{q}\right\}$ be the norm completion of $\mathscr{C}_{*}(X)$. Thus $C_{q}^{l_{1}}(X)=$ $\left\{\sum_{i=1}^{\infty} a_{i} \sigma_{i}\left|\sum_{i=1}^{\infty}\right| a_{i} \mid<\infty\right\}$ is a Banach space. Passing to the dual Banach spaces, we obtain a cochain complex $\mathscr{C}_{b}^{*}(X)=\left\{C_{b}^{q}(X), \delta_{q}\right\}$. It is a subcomplex of the ordinary singular cochain complex consisting of bounded cochains. The homology of $\mathscr{C}_{*}^{I_{k}}(X)$, denoted by $H_{*}^{l_{1}}(X)$, is called $l_{1}$ homology of $X$ and the cohomology of $\mathscr{C}_{b}^{*}(X)$, denoted by $H_{b}^{*}(X)$, is called bounded cohomology of $X$. The inclusions induce homomorphisms $H_{*}(X) \rightarrow H_{*}^{l_{1}}(X)$ and $H_{b}^{*}(X) \rightarrow H^{*}(X)$.

Since the image of a bounded operator is not necessarily a closed subspace, it may happen that the pseudonorms induced on $H_{*}^{l_{1}}(X)$ or $H_{b}^{*}(X)$ are not norms. Following Mitsumatsu [5], we define $\bar{H}_{*}^{l_{1}}(X)$ (resp. $\left.\bar{H}_{b}^{*}(X)\right)$ to be the quotient of $H_{*}^{l_{1}}(X)$ (resp. $H_{b}^{*}(X)$ ) by the subspace of pseudonorm zero. In other words, $\bar{H}_{q}^{l_{1}}(X)=Z_{q}^{l_{1}}(X) / \bar{B}_{q}^{l_{1}}(X)$ and $\bar{H}_{b}^{q}(X)=Z_{b}^{q}(X) / \bar{B}_{b}^{q}(X)$, where $Z$ or $B$ denotes the spaces of (co)cycles or (co)boundaries of the corresponding complex and $\bar{B}$ denotes the closure of $B$. Notice that $\bar{H}_{q}^{l_{1}}(X)$ and $\bar{H}_{b}^{q}(X)$ are Banach spaces. There is a

Received by the editors July $19,1984$.

1980 Mathematics Subject Classification. Primary 55N99; Secondary 57T99.

Key words and phrases. Bounded cohomology, $l^{1}$ homology, groups of homeomorphisms.

(C)1985 American Mathematical Society $0002-9939 / 85 \$ 1.00+\$ .25$ per page 
surjective homomorphism $\bar{H}_{b}^{q}(X) \rightarrow\left(\bar{H}_{q}^{l_{1}}(X)\right)^{\prime}$, where' denotes the dual Banach space.

Now for a group $G$, starting from the real chain complex $\mathscr{C}_{*}(G)=\left\{C_{q}(G), \delta_{q}\right\}$, similar constructions as above are made, yielding $l^{1}$ homology $H_{*}^{l_{1}}(G)$ and bounded cohomology $H_{b}^{*}(G)$ of $G$.

2. Uniform boundary condition. A chain complex is called normed if each chain group is a normed linear space over $\mathbf{R}$ and each differential is a bounded linear operator.

DEFINITION 2.1. A normed chain complex $\mathscr{C}_{*}$ is said to satisfy $q$ uniform boundary condition ( $q$-UBC, for short) if there exists a number $K>0$ such that for any boundary $z \in B_{q}$, there is a chain $c \in C_{q+1}$ satisfying $\partial c=z$ and $\|c\| \leqslant K\|z\|$.

Definition 2.2. (i) A topological space or a group is said to satisfy $q-\mathrm{UBC}$ if its ordinary chain complex satisfies $q$-UBC.

(ii) It is said to satisfy $q$-UBC ${ }^{l_{1}}$ if its $l^{1}$ chain complex satisfies $q$-UBC.

THEOREM 2.3. For spaces or groups, the following conditions are equivalent:

(i) $q-\mathrm{UBC}^{l_{1}}$.

(ii) $B_{q}^{l_{1}}$ is closed in $C_{q}^{l_{1}}$.

(iii) $\bar{H}_{q}^{l_{1}}=H_{q}^{l_{1}}$.

(iv) $\bar{H}_{b}^{q+1}=H_{b}^{q+1}$.

(v) The surjective homomorphism $H_{b}^{q+1} \rightarrow\left(\bar{H}_{q+1}^{l_{1}}\right)^{\prime}$ is injective.

Proof. (i) is clearly equivalent to that the bijection $C_{q+1}^{l_{1}} / Z_{q+1}^{l_{1}} \rightarrow B_{q}^{l_{1}}$ has a bounded inverse, that is, they are homeomorphic. Since $C_{q+1}^{l_{1}} / Z_{q+1}^{l_{1}}$ is a Banach space, it follows that $B_{q}^{l_{1}}$ is also a Banach space and hence (ii) holds. Conversely if (ii) is satisfied, then $B_{q}^{l_{1}}$ is a Banach space and the open mapping theorem (cf. [11]) applied to the above bijection implies (i).

(ii) $\Leftrightarrow$ (iii) is clear.

(iii) $\Leftrightarrow$ (iv) is a direct consequence of the closed range theorem.

(i) $\Rightarrow(\mathrm{v})$. Take $f \in Z_{b}^{q+1}$ so that $f(z)=0$ for all $z \in Z_{q+1}^{l_{1}}$. By (i), the map $B_{q}^{l_{1}} \leftarrow C_{q+1}^{l_{1}} / Z_{q+1}^{l_{1}} \stackrel{\dot{f}}{\rightarrow} \mathbf{R}$ is bounded and thus by the Hahn-Banach theorem has an extension to $C_{q}^{l_{1}}$. This shows (v).

$(\mathrm{v}) \Rightarrow$ (iv). It suffices to show $\operatorname{Ker}\left(H_{b}^{q+1} \rightarrow \bar{H}_{b}^{q+1}\right) \subset \operatorname{Ker}\left(H_{b}^{q+1} \rightarrow\left(\bar{H}_{q+1}^{l_{1}}\right)^{\prime}\right)$. Let $f \in \bar{B}_{b}^{q+1}$. Then $f=\lim _{i \rightarrow \infty} f_{i}\left(f_{i} \in B_{b}^{q+1}\right)$. Thus for any $z \in Z_{q+1}^{l_{1}}$, we have $f(z)=$ $\lim _{i \rightarrow \infty} f_{i}(z)=0$. This completes the proof. Q.E.D.

COROLlaRY 2.4. (i) If $\bar{H}_{q}^{l_{1}}=H_{q}^{l_{1}}$ and $\bar{H}_{q+1}^{l_{1}}=0$, then $H_{b}^{q+1}=0$.

(ii) If $\bar{H}_{b}^{q+1}=H_{b}^{q+1}$ and $\bar{H}_{b}^{q}=0$, then $H_{q}^{l_{1}}=0$.

(iii) The reduced bounded cohomology $\tilde{H}_{b}^{*}$ vanishes if and only if the reduced $l^{1}$ homology $\tilde{H}_{*}^{l_{1}}$ also vanishes.

In Brooks [1] and Gromov [2], it is shown that the reduced bounded cohomology vanishes for spaces with amenable $\pi_{1}$. This, combined with the above corollary, gives

Corollary 2.5. If $\pi_{1}(X)$ is amenable, then $\tilde{H}_{*}^{l_{1}}(X)=0$. 
ReMARK 2.6. It seems plausible that the $l^{1}$ homology of a space depends only on its fundamental group. But we do not have a proof.

COROLLARY 2.7. For any space or a group, we have

(i) $H_{1}^{I_{1}}=H_{b}^{1}=0$.

(ii) $H_{b}^{-2}=H_{b}^{2}$. Equivalently, $H_{b}^{2}$ is a Banach space.

Proof. First we deal with a group $G$. In [5], Mitsumatsu constructed for $g \in g$,

$$
S(g)=\sum_{k=1}^{\infty} \frac{1}{2^{k}}\left(g^{k}, g^{k}\right) \in C_{2}^{l_{1}}(G) \text {. }
$$

Clearly, $\|S(g)\|=1$ and $\partial S(g)=g$, showing that $H_{1}^{l_{1}}(G)=0$ and that $G$ satisfies 1-UBC ${ }^{l_{1}}$. From this follows the corollary. Notice that $0-\mathrm{UBC}^{l_{1}}$ is always satisfied.

For spaces, according to Gromov [2], $H_{b}^{2}(X)$ is isometric to $H_{b}^{2}\left(\pi_{1}(X)\right)$. This shows (ii). Also it is known that $H_{b}^{1}(X)=0$ [2]. Thus (i) follows from Corollary 2.4. Q.E.D.

We have shown that $1-\mathrm{UBC}^{l_{1}}$ is always true. It would be interesting to determine whether $q$-UBC ${ }^{l_{1}}$ always holds or not.

Next, we investigate $q$-UBC for spaces or groups.

THEOREM 2.8. The following conditions are equivalent:

(i) $q$-UBC.

(ii) $q$ - $\mathrm{UBC}^{l_{1}}$ and $Z_{q+1}$ is dense in $Z_{q+1}^{l_{1}}$.

(iii) The homomorphism $H_{b}^{q+1} \rightarrow H^{q+1}$ is injective.

Proof. (i) $\Rightarrow$ (ii). We first prove $q-\mathrm{UBC}^{l_{1}}$. Clearly $B_{q}$ is dense in $B_{q}^{l_{1}}$. Further a standard argument shows that for any $z \in B_{q}^{l_{1}}$, there exist $z_{i} \in B_{q}$ such that $\sum_{i=1}^{\infty} z_{i}=z$ and $\sum_{i=1}^{\infty}\left\|z_{i}\right\| \leqslant(1+\varepsilon)\|z\|$. Now by $q$-UBC, one can choose $c_{i} \in C_{q+1}$ such that $\partial c_{i}=z_{i}$ and $\left\|c_{i}\right\| \leqslant K\left\|z_{i}\right\|$. Let $c=\sum_{i=1}^{\infty} c_{i} \in C_{q+1}^{l_{1}}$. Then $\partial c=z$ and $\|c\| \leqslant(1+\varepsilon) K\|z\|$.

Next we prove that $Z_{q+1}$ is dense in $Z_{q+1}^{l_{1}}$. Take $z \in Z_{q+1}^{l_{1}}$ and let $z=\lim _{i \rightarrow \infty} c_{i}$ $\left(c_{i} \in C_{q+1}\right)$. Choose an element $d_{i} \in C_{q+1}$ such that $\partial d_{i}=-\partial c_{i}$ and $\left\|d_{i}\right\| \leqslant K\left\|\partial c_{i}\right\|$. Then we have

$$
\left\|\partial c_{i}\right\|=\left\|\partial\left(c_{i}-z\right)\right\| \leqslant\|\partial\|\left\|c_{i}-z\right\|=(q+2)\left\|c_{i}-z\right\| .
$$

Hence $\left\|d_{i}\right\| \leqslant(q+2) K\left\|c_{i}-z\right\|$. Now $c_{i}+d_{i} \in Z_{q+1}$ and $c_{i}+d_{i} \rightarrow z$.

(ii) $\Rightarrow$ (i). This is left to the reader.

(ii) $\Rightarrow$ (iii). Take $f \in Z_{b}^{q+1}$ such that $[f]=0$ in $H^{q+1}$, that is, $f(z)=0$ for any $z \in Z_{q+1}$. Then $f(z)=0$ for any $z \in Z_{q+1}^{l_{1}}$. Thus $f$ induces a bounded map $\bar{f}$ : $C_{q+1}^{l_{1}} / Z_{q+1}^{l_{1}} \rightarrow \mathbf{R}$. Now $q-\mathrm{UBC}^{l_{1}}$ implies that the bijection $C_{q+1}^{l_{1}} / Z_{q+1}^{l_{1}} \rightarrow B_{q}^{l_{1}}$ has a bounded inverse. Compose it with $\bar{f}$ and extend to the whole of $C_{q}^{l_{1}}$ by the Hahn-Banach theorem. This shows (iii).

(iii) $\Rightarrow$ (ii). Notice that the map $H_{b}^{q+1} \rightarrow H^{q+1}$ is a composite of maps $H_{b}^{q+1} \rightarrow$ $\bar{H}_{b}^{q+1} \rightarrow H^{q+1}$. Injectivity of the first map implies $q-\mathrm{UBC}^{l_{1}}$ by Theorem 2.3. Next, clearly (iii) implies that the image of the map $H_{q+1} \rightarrow \bar{H}_{q+1}^{l_{1}}$ is dense. From this the denseness of $Z_{q+1}$ follows easily. Q.E.D. 
Definition 2.9. A group $G$ is said to be uniformly perfect if for some $N>0$, any $g \in G$ can be represented as a product of at most $N$ commutators.

LEMMA 2.10. If a group $G$ is uniformly perfect, then it satisfies 1-UBC.

Proof. Notice that for $f_{i}, g, h \in G$,

$$
\begin{gathered}
\partial\left(\left(f_{1}, f_{2}\right)+\left(f_{1} f_{2}, f_{3}\right)+\cdots+\left(f_{1} f_{2} \cdots f_{N-1}, f_{N}\right)\right) \\
=\left(f_{1}\right)+\left(f_{2}\right)+\cdots+\left(f_{N}\right)-\left(f_{1} f_{2} \cdots f_{N}\right)
\end{gathered}
$$

and

$$
\partial\left(([g, h], h)+\left(g h g^{-1}, g\right)-(g, h)\right)=([g, h])
$$

where ( ) denotes a chain and [ ] a commutator. This shows for all $f \in G$ there exists $c \in C_{2}(G)$ such that $\partial c=(f)$ and $\|c\| \leqslant 4 N-1$. That is, $G$ satisfies 1-UBC. Q.E.D.

Corollary 2.11. If $G$ is uniformly perfect, then the map $H_{b}^{2}(G) \rightarrow H^{2}(G)$ is injective.

As applications, we shall compute $H_{b}^{2}$ for some groups.

EXAMPLE 2.12. The group of all orientation preserving homeomorphisms of $S^{1}$, denoted by $\mathrm{Homeo}_{+}\left(S^{1}\right)$, is uniformly perfect. In fact any element is a product of two homeomorphisms with compact support, which are commutators by Mather [4]. Thus $H_{b}^{2}$ injects into $H^{2}$. Now it is a consequence of Thurston's general result [8] that $H^{*}\left(\right.$ Homeo $\left._{+}\left(S^{1}\right) ; \mathbf{Z}\right) \cong \mathbf{Z}[\chi]$, where $\chi \in H^{2}$ is the Euler class. Now $H_{b}^{*}$ is mapped onto real cohomology, because $\chi$ can be represented by a bounded cocycle (see Morita [6]). Hence we have $H_{b}^{2}\left(\operatorname{Homeo}_{+}\left(S^{1}\right)\right) \cong \mathbf{R}$.

EXAMPLE 2.13. Sah and Wagoner [7] have calculated second homology of certain Lie groups (considered to be discrete groups). Combined with our result, this gives information about $H_{b}^{2}$. For example, $\mathrm{SL}_{2} \mathbf{R}$ is uniformly perfect (see Wood [10]) and $H_{2}\left(\mathrm{SL}_{2} \mathbf{R} ; \mathbf{Z}\right)$ is isomorphic to $\mathbf{Z} \oplus A$, where $A$ is a certain $\mathbf{Q}$-vector space. $\mathbf{Z}$ is detected by the "volume class" $\in H^{2}\left(\mathrm{SL}_{2} \mathbf{R}\right)$, which is a bounded cohomology class. Any element of $A$ is supporied on a torus (see [7] and Tsuboi [9]). From these, we can conclude $H_{b}^{2}\left(\mathrm{SL}_{2} \mathbf{R}\right) \cong H_{b}^{2}\left(\mathrm{PSL}_{2} \mathbf{R}\right) \cong \mathbf{R}$.

3. Bounded cohomology of $\mathrm{Homeo}_{K}\left(\mathbf{R}^{n}\right)$. In this section we prove the vanishing of bounded cohomology and $l^{1}$ homology of $\mathrm{Homeo}_{K}\left(\mathbf{R}^{n}\right)$, the group of all homeomorphisms of $\mathbf{R}^{n}$ with compact support.

THEOREM 3.1. For $q>0$,

$$
H_{b}^{q}\left(\operatorname{Homeo}_{K}\left(\mathbf{R}^{n}\right)\right)=H_{q}^{I_{1}}\left(\operatorname{Homeo}_{K}\left(\mathbf{R}^{n}\right)\right)=0 .
$$

Our argument is a refinement of Mather's proof of the acyclicity of $\mathrm{Homeo}_{K}\left(\mathbf{R}^{n}\right)$. In the sequel we follow Mather [4]. We write $G=\operatorname{Homeo}_{K}\left(\mathbf{R}^{n}\right)$ and $G^{i}=\{g \in G$ : supp $g \subset$ Int $\left.i D^{n}\right\}(i=1,2,3)$, where $D^{n}$ is the unit ball. Inclusions are denoted by $\iota^{1}: G^{1} \rightarrow G^{2}, \iota^{2}: G^{2} \rightarrow G^{3}, i=\iota^{2} \iota^{1}$ and $\iota: G^{1} \rightarrow G$. Let $C_{q}$ and $C_{q}^{i}$ be the chain complex of $G$ and $G^{i} . Z_{q}$ and $Z_{q}^{i}$ (resp. $B_{q}$ and $B_{q}^{i}$ ) denote the cycle group (resp. 
boundary group) of the corresponding complexes. In fact, $Z_{q}=B_{q}$ and $Z_{q}^{i}=B_{q}^{i}$ by the acyclicity of the group.

We shall prove inductively the existence of bounded linear operators $S_{q}: B_{q}^{1} \rightarrow C_{q+1}$ such that $\partial_{q+1} S_{q}=\iota_{*}$. Let us show first that this suffices for our purpose. We have only to show $q$-UBC for $G$, because the acyclicity of $G$, together with Theorem 2.8 and Corollary 2.4 yields Theorem 3.1. Take $z \in B_{q}^{1}$. Choose $\varphi \in G$ such that $\varphi$ is the identity on $\operatorname{supp} z$ and maps $\operatorname{supp} S_{q}(z)$ into Int $D^{n}$. Define $I_{\varphi}: G \rightarrow G$ by $I_{\varphi}(g)=$ $\varphi g \varphi^{-1}$. Then we have $I_{\varphi^{*}}\left(S_{q}(z)\right) \in C_{q+1}^{1},\left\|I_{\varphi^{*}}\left(S_{q}(z)\right)\right\|=\left\|S_{q}(z)\right\| \leqslant\left\|S_{q}\right\|\|z\|$ and $\partial\left(I_{\varphi^{*}}\left(S_{q}(z)\right)\right)=I_{\varphi^{*}}\left(\partial S_{q}(z)\right)=I_{\varphi^{*}}(z)=z$. This proves $q$-UBC for $G^{1}$, hence for $G$.

$S_{1}$ is constructed in an elementary fashion as follows. Choose $k \in G$ such that $k\left(3 D^{n}\right) \cap 3 D^{n}=\varnothing$ and that $k^{i}\left(3 D^{n}\right)$ tends to one point as $i \rightarrow \infty$. Define $\psi_{1}$ : $G^{3} \rightarrow G$ by $\psi_{1}(g)=\sum_{i=1}^{\infty} k^{i} g k^{-i}$ and let $\psi_{0}(g)=k^{-1} \psi_{1}(g) k$. Then for any $g \in G^{3}$, supp $g \cap \operatorname{supp} \psi_{1}(g)=\varnothing$ and $\psi_{0}(g)=g \psi_{1}(g)$. The restrictions of $\psi_{i}$ to $G^{1}$ are denoted by the same letter. Now we define a bounded linear map $S_{1}: B_{1}^{1} \rightarrow C_{2}$ by

$$
S_{1}(g)=(k, g)-\left(\psi_{1}(g), k\right)+\left(k g, \psi_{1}(g)\right) \text {. }
$$

Direct computation shows $\partial_{2} S_{1}=\iota_{*}$.

Next we assume there exist $S_{j}^{1}: B_{j}^{1} \rightarrow C_{j+1}^{2}$ and $S_{j}^{2}: B_{j}^{2} \rightarrow C_{j+1}^{3}$ for $0 \leqslant j \leqslant q-1$ and construct $S_{q}: B_{j}^{1} \rightarrow C_{j+1}$. Let $\alpha: C_{*}(G \times G) \rightarrow C_{*} \otimes C_{*}\left(\right.$ resp. $\beta: C_{*} \otimes C_{*} \rightarrow$ $\left.C_{*}(G \times G)\right)$ be the Alexander-Whitney map (resp. Eilenberg-Mac Lane map) (see Mac Lane [3]). Those maps for $G^{i}$ are also denoted by the same letters. They are functorial and if we give a norm to $C_{*} \otimes C_{*}$ in a canonical manner, they are bounded linear.

For each $z \in B_{q}^{1}$, define $D(z)=\alpha \Delta_{*} z-(z \otimes 1+1 \otimes z)$, where $\Delta: G^{1} \rightarrow$ $G^{1} \times G^{1}$ is the diagonal map. Then $D(z) \in Z_{q}^{\prime}\left(C^{1} \otimes C^{1}\right)=Z_{q}\left(C^{1} \otimes C^{1}\right) \cap$ $\sum_{i=1}^{q-1} C_{i}^{1} \otimes C_{q-}^{1} . D$ is bounded linear. Now let $Z^{1}$ and $\bar{B}^{1}$ be the chain complexes (with trivial differential) defined by $\left(Z^{1}\right)_{q}=Z_{q}^{1}$ and $\left(\bar{B}^{1}\right)_{q}=B_{q-1}^{1}$. Then we have the following commutative diagram, whose horizontal sequences are all exact.

$$
\begin{aligned}
& 0 \rightarrow\left(C^{1} \otimes Z^{1}\right)_{q+1} \quad \rightarrow \quad\left(C^{1} \otimes C^{1}\right)_{q+1} \stackrel{1 \otimes \partial}{\rightarrow} \quad\left(C^{1} \otimes \bar{B}^{1}\right)_{q+1} \quad \rightarrow \quad 0 \\
& \downarrow \partial \quad \downarrow \partial \quad \downarrow \partial \\
& 0 \quad \rightarrow \quad\left(C^{1} \otimes Z^{1}\right)_{q} \quad \rightarrow \quad\left(C^{1} \otimes C^{1}\right)_{q} \quad \stackrel{1 \otimes \partial}{\rightarrow} \quad\left(C^{1} \otimes \bar{B}^{1}\right)_{q} \quad \rightarrow \quad 0 \\
& \downarrow \partial \quad \downarrow \partial \quad \downarrow \partial \\
& 0 \quad \rightarrow \quad\left(C^{1} \otimes Z^{1}\right)_{q-1} \quad \rightarrow \quad\left(C^{1} \otimes F^{1}\right)_{q-1} \stackrel{1 \otimes \partial}{\rightarrow} \quad\left(C^{1} \otimes \bar{B}^{1}\right)_{q-1} \quad \rightarrow \quad 0
\end{aligned}
$$

Analogous diagrams are considered for $G^{2}$ and $G^{3}$. They are combined by $\iota_{*}^{1}$ and $\iota_{*}^{2}$. Now because $\partial(D(z))=0,(1 \otimes \partial) D(z)$ is contained in $Z_{q}^{\prime}\left(C^{1} \otimes \bar{B}^{1}\right)$. Notice that $Z_{q}^{\prime}\left(C \otimes \bar{B}^{1}\right)=\left(Z^{1} \otimes \bar{B}^{1}\right)_{q}^{\prime}$. Hence by the induction assumption we can consider

$$
\left(S^{1} \otimes S^{1}\right)(1 \otimes \partial) D(z)=\left(S^{1} \otimes S^{1} \partial\right) D(z) \in\left(C^{2} \otimes C^{2}\right)_{q+1} \text {. }
$$

Let $u=\left(\iota_{*}^{1} \otimes \iota_{*}^{1}\right) D(z)-\partial\left(S^{1} \otimes S^{1} \partial\right) D(z) \in\left(C^{2} \otimes C^{2}\right)_{q}$. Direct computation shows that $(1 \otimes \partial) u=0$. Thus we have $u \in\left(C^{2} \otimes Z^{2}\right)_{q}^{\prime}$. Also we have $\partial u=0$. That is,

$$
u \in Z_{q}^{\prime}\left(C^{2} \otimes Z^{2}\right)=\left(Z^{2} \otimes Z^{2}\right)_{q}^{\prime}
$$


Therefore by the induction assumption, we can consider

$$
\left(S^{2} \otimes\left(\iota_{*}^{2}-S^{2} \partial\right)\right) u \in\left(C^{3} \otimes C^{3}\right)_{q+1} .
$$

Again direct computation shows that

$$
\partial\left(S^{2} \otimes\left(\iota_{*}^{2}-S^{2} \partial\right)\right) u=\left(\iota_{*}^{2} \otimes \iota_{*}^{2}\right) u
$$

Hence we have $\left(i_{*} \otimes i_{*}\right) D(z)=\partial(E D(z))$, where

$$
E=\left(\iota_{*}^{2} S^{1} \otimes \iota_{*}^{2} S^{1} \partial\right)+\left(S^{2} \otimes\left(\iota_{*}^{2}-S^{2} \partial\right)\right)\left(\iota_{*}^{1}-\partial\left(S^{1} \otimes S^{1} \partial\right)\right) .
$$

$E: Z_{q}^{\prime}\left(C^{1} \otimes C^{1}\right) \rightarrow\left(C^{3} \otimes C^{3}\right)_{q+1}$ is a bounded linear map. We can now write

$$
\left(i_{*} \otimes i_{*}\right) \alpha \Delta_{*} z=\left(i_{*} \otimes i_{*}\right) z \otimes 1+\partial(E D(z))+\left(i_{*} \otimes i_{*}\right)(1 \otimes z) .
$$

Let $\eta: G^{3} \otimes G^{3} \rightarrow G$ be the homomorphism given by $\eta(g, h)=g \psi_{1}(h)$. Applying $\eta_{*} \beta$, we get

$$
\eta_{*}(i \times i)_{*} \beta \alpha \Delta_{*} z=\iota_{*} z+\eta_{*} \beta \partial(E D(z))+\chi_{1 *} z
$$

As is well known, $\beta \alpha$ is chain homotopic to the identity. Namely there is a linear map $\Phi: C_{*}\left(G^{1} \times G^{1}\right) \rightarrow C_{*+1}\left(G^{1} \times G^{1}\right)$ such that $\beta \alpha-\mathrm{id}=\partial \Phi+\Phi$. It is easy to show that we can choose $\Phi$ as a bounded linear map. Now $\beta \alpha \Delta_{*} z=\Delta_{*} z+$ $\partial \Phi\left(\Delta_{*} z\right)$. Because $\eta \Delta=\psi_{0}$, we have

$$
\psi_{0^{*}} z+\eta_{*}(i \times \bar{\imath})_{*} \partial \Phi \Delta_{*} z=\iota_{*} z+\eta_{*} \beta \partial E D(z)=\psi_{1^{*}} z
$$

Two homomorphisms $\psi_{0}$ and $\psi_{1}$ are conjugate and thus $\psi_{0^{*}}$ is chain homotopic to $\psi_{1^{*}}$. Namely there is a linear map $\Theta: C_{*}^{1} \rightarrow C_{*+1}$ such that $\psi_{1^{*}}-\psi_{0^{*}}=\partial \Theta+\Theta \partial$. Here we can also choose $\Theta$ to be bounded linear. Finally we obtain $\iota_{*}=\partial_{q+1} S_{q}$, where

$$
S_{q}=\eta_{*}(i \times \bar{\imath})_{*} \Phi \Delta_{*}-\Theta-\eta_{*} \beta E D .
$$

This completes the proof.

\section{REFERENCES}

1. R. Brooks, Some remarks on bounded cohomology, Ann. of Math. Stud., no. 97, Princeton Univ. Press, Princeton, N. J., 1981, pp. 53-63.

2. M. Gromov, Volume and bounded cohomology, Inst. Hautes Études Sci. Publ. Math. 56 (1982), 5-99.

3. S. Mac Lane, Homology, Springer-Verlag, Berlin and New York, 1963.

4. J. Mather, The vanishing of homology of certain groups of homeomorphisms, Topology 10 (1973), 297-298.

5. Y. Mitsumatsu, Bounded cohomology and $l^{1}$ homology of surfaces, Topology (to appear).

6. S. Morita, Characteristic classes of surface bundles. I, preprint.

7. C-H. Sah and J. B. Wagoner, Second homology of Lie groups made discrete, Comm. Algebra 5 (1977), $611-642$.

8. W. Thurston, Foliations and groups of diffeomorphisms, Bull. Amer. Math. Soc. 80 (1974), 304-307.

9. T. Tsuboi, Foliated cobordism classes of certain foliated $S^{1}$-bundles over surfaces, Topology 23 (1984), $233-244$.

10. J. Wood, Bundles with totally discontinuous structure group, Comm. Math. Helv. 46 (1971), 257-273.

11. K. Yosida, Functional analysis, Springer-Verlag, Berlin and New York, 1960.

Department of Mathematics, College of Science and Technology, Nihon University, 101 Kanda - Surugadai, Chiyoda - KU, TOKyo, JaPAN

Department of Mathematics, College of General Education, Tokyo University, 153 Komaba, MEGURO - KU, TOKYO, JAPAN 